\title{
How effective are common medications: a perspective based on meta-analyses of major drugs
}

\author{
Stefan Leucht ${ }^{{ }^{*}{ }^{+}}$, Bartosz Helfer ${ }^{1 \dagger}$, Gerald Gartlehner ${ }^{2,3}$ and John M. Davis ${ }^{4}$
}

\begin{abstract}
The vastness of clinical data and the progressing specialization of medical knowledge may lead to misinterpretation of medication efficacy. To show a realistic perspective on drug efficacy we present meta-analyses on some of the most commonly used pharmacological interventions. For each pharmacological intervention we present statistical indexes (absolute risk or response difference, percentage response ratio, mean difference, standardized mean difference) that are often used to represent efficacy. We found that some of the medications have relatively low effect sizes with only 11 out of 17 of them showing a minimal clinically important difference. Efficacy was often established based on surrogate outcomes and not the more relevant patient-oriented outcomes. As the interpretation of the efficacy of medication is complex, more training for physicians might be needed to get a more realistic view of drug efficacy. That could help prevent harmful overtreatment and reinforce an evidencebased, but personalized medicine.
\end{abstract}

Keywords: Absolute risk or response difference, Common medications, Drug classes, Drug efficacy, Mean difference, Medication efficacy, Meta-analysis, Percentage response ratio, Pharmacological interventions, Standardized mean difference, Schizophrenia, Depression

\section{Background}

Medicine is becoming so highly specialized and the clinical literature is growing so fast, that few doctors let alone the lay public have a working knowledge of the detailed evidence on drugs outside their specialty [1]. This is despite the fact that clinicians must often evaluate comparative risks and benefits of treatments for patients with multiple maladies. Studies show that decision making can be distorted by various cognitive biases such as a physician's tendency to remember dramatically successful cases and forget ones that failed or to misinterpret the statistical indices used in clinical trials and meta-analyses [2]. This may lead the physician to overestimate the efficacy of treatments, which in turn may be one of the causes of harmful overtreatment [3].

\footnotetext{
* Correspondence: Stefan.Leucht@|rz.tu-muenchen.de

${ }^{\dagger}$ Equal contributors

'Department of Psychiatry and Psychotherapy, Technische Universität München, Klinikum rechts der Isar, Ismaninger Straße 22, 81675 Munich, Germany

Full list of author information is available at the end of the article
}

\section{Common pharmacological treatments}

We would like to present a realistic perspective on the general efficacy of common pharmacological treatments. Following the general methods of a previous overview of reviews [4], we identified systematic reviews of randomized controlled trials with meta-analysis comparing drugs used in specific therapy types with placebo. We included 20 most common therapy types as measured by the number of on-therapy patients in the US, according to the IMS Institute for Healthcare Informatics [5]. For each therapy type listed there we identified primary pharmacological treatments and their primary indications (as suggested by the IMS review and verified by national and international treatment guidelines). Then using PubMed we searched (last search: 5 August 2014, see Additional file 1) for the broadest and most recent meta-analysis on that treatment. If possible, we included meta-analyses on monotherapy rather than combination therapy, on all patients rather than a sub-group of patients (for example, we preferred reviews on all age groups, over ones restricted to adults or children) and 
on broad drug classes rather than narrow ones or single drugs (for example, we preferred a meta-analysis on all antihypertensive drugs, over ones on ACE inhibitors or enalapril). If a meta-analysis on the whole therapy type (for example, any narcotic) was not available, we included a frequently used example (for example, oxycodone + paracetamol, which is the most frequently used painkiller according to the IMS report for which we found a meta-analysis fulfilling our inclusion criteria). For a more detailed description of our methods, please refer to the protocol (see Additional file 2).

\section{Measures of medication efficacy}

Figure 1 lists examples of medications used primarily in the 20 most common therapy types together with a number of statistical indices. Here we explain how these measures are calculated and give some examples:

1) Absolute risk or response difference (ARD) is the risk or percentage of responders in group $B$ subtracted from the risk or percentage of responders in group A. For example, mortality was $2 \%$ for drug treatment and $4 \%$ for placebo, which gives an $\mathrm{ARD}=|-2 \%|$. For responder rates, if $45 \%$ of patients responded in the drug group and $30 \%$ in the placebo group, the ARD is $15 \%$.

2) Percentage response ratio (PRR) is the percentage of responders in group A divided by the percentage responders in group B. For example, if $45 \%$ of participants responded to drug treatment in group $\mathrm{A}$ and $30 \%$ to placebo in group B, the PRR is $50 \%$, because $0.45 / 0.3=1.5$. This means that there were $50 \%$ more responders in group A compared to group B.

3) Mean difference (MD) is the mean from group $B$ subtracted from the mean in group A. For example, if the mean total sleep time at the end of treatment in the drug group was 5 hours and 10 minutes and in the placebo group 4 hours and 55 minutes, the MD is 15 minutes.

4) Standardized mean difference (SMD) is the mean from group B subtracted from the mean in group A and divided by the pooled standard deviation (SD). For example, if the average weight of participants at the end of treatment was $79 \mathrm{~kg}$ in the drug group and $83 \mathrm{~kg}$ in the placebo group and the pooled SD was $8 \mathrm{~kg}$, the SMD is 0.5 .

Effect sizes at Fig. 1 are expressed graphically as SMDs and are ranked as "small" (0.2), "medium" (around 0.5) or "large" (above 0.8) [6]. We also present the percentage of responders in the drug and placebo group and, if appropriate, the number of trials $(\mathrm{N})$ and patients $(\mathrm{n})$ for each meta-analysis, as well as the AMSTAR score, which is a measure of methodological quality of systematic reviews [7].

\section{The efficacy of common medications}

Differences larger than one standard deviation (that is, SMD >1) between the drug and placebo groups are uncommon, examples being proton pump inhibitors for reflux esophagitis [8] or oxycodone plus paracetamol for postoperative pain [9]. For many other medications the effect sizes were much smaller. For example, antihypertensive drugs reduced systolic and diastolic blood pressure by only $10 \mathrm{mmHg}$ and $5 \mathrm{mmHg}$, respectively [10], the ARD between aspirin and placebo for primary prevention of cardiovascular events was only $0.07 \%$ per year [11], and the ARD for antidepressants and placebo for major depressive disorder was $17 \%$ [12].

For an outcome affecting quality of life, $1 / 2$ of a standard deviation is considered to be a minimal clinically important difference [13]. Out of 17 common pharmacological treatments examined, only 11 met this threshold. In four of them efficacy was represented by surrogate outcomes, such as diastolic blood pressure or fasting plasma glucose, and not patient-oriented outcomes, such as pain, mortality or adverse events. Therefore, patients might not have experienced substantial benefits related to their well-being and quality of life after therapy with some of these drugs. Moreover many of the included meta-analyses had a low methodological quality as represented by median AMSTAR score of 7/11 (interquartile range 5 to 9 ).

\section{Surrogate outcomes versus patient-oriented outcomes}

Figure 1 also illustrates that surrogate outcomes often show dramatic effects, while the effects on patientoriented outcomes are much smaller. For example, statins reduce cholesterol by $30 \%$ on average [14]. However, high cholesterol alone does not directly produce pain or disability. For long-term consequences, such as cardiovascular events and mortality, the effects are smaller (ARD between statins and placebo of $4 \%$ for cardiovascular events and $1.2 \%$ for mortality within 5 years [15]). In hypertension, medium effect sizes for reductions of hypertension [10] lead to comparatively small reductions of cardiovascular events [16], and metformin strongly reduces glucose [17], but there is no evidence of a reduction in mortality [18]. Among the seven outcomes that can be both objectively measured and are patient-oriented (marked in red color in Fig. 1) only one shows a big effect size (remission of reflux esophagitis by proton pump inhibitors [8]).

\section{Statistical indices can be misleading}

In general, relative risk reductions suggest larger differences than ARDs. For example, statins reduced the 
Therapy type

Proton pump inhibitors for reduction of gastric acid [ 8 ] Remission of reflux oesophagitis $\mathrm{PL}=28 \%, \mathrm{D}=83 \%, \mathrm{ARD}=58 \%, \mathrm{PRR}=256 \%, \mathrm{~N}=5, \mathrm{n}=645$

Oxycodone + paracetamol for pain relief [ 9 ] $50 \%$ postoperative pain reduction in $4-6 \mathrm{~h}$ $P L=14 \%, D=51 \%, A R D=26 \%, P R R=290 \%, N=10, n=1043$

Tiotropium for chronic obstructive pulmonary disease [30] Forced expiratory volume in one second in liters $\mathrm{MD}=0.2 \mathrm{l}, \mathrm{N}=4, \mathrm{n}=1735$

Levodopa for Parkinson's disease [32] Reduction of symptoms $\mathrm{MD}=7, \mathrm{~N}=1, \mathrm{n}=311$

Metformin for type 2 diabetes [17] Fasting glucose $(\mathrm{mmol} / \mathrm{l})$ $M D=1.84, N=12, n=1587$

Metformin for type 2 diabetes [18]

$\mathrm{PL}=6 \%, \mathrm{D}=2.7 \%, \mathrm{ARD}=0 \%, \mathrm{PRR}=95 \%, \mathrm{~N}=11, \mathrm{M}=12840$

Sumatriptan for migraine [31] Pain free after 2 hours

$\% P L=11, \% D=32, A R D=23 \%, P R R=320 \%, N=16, n=6571$

Methylphenidates for attention deficit hyperactivity disorder [24] Reduction of symptoms $\mathrm{N}=22, \mathrm{n}=2856$

Benzodiazepines for insomnia [25] Sleep onset latency $\mathrm{MD}=14$ minutes, $\mathrm{N}=8, \mathrm{n}=539$

Antihypertensives for hypertension [10] RR systolic $(\mathrm{mmHg})$ $M D=9.4, N=94, n=17641$

Angiotensin-converting-enzyme-inhibitors for hypertension [16] Cardiovascular events

$P L=18 \%, D=14 \%, A R D=4 \%, P R R=22 \%, N=5, n=18229$

Corticosteroids for chronic asthma [29] forced expiratory volume in one second in liters $\mathrm{MD}=0.31, \mathrm{~N}=19, \mathrm{n}=3271$

Antipsychotics for schizophrenia [26] Reduction of symptoms $\mathrm{N}=35, \mathrm{n}=5568$

Cholinesterase inhibitors for Alzheimer's disease [23] Reduction of symptoms $M D=2.38, N=10, n=4236$

Bisphosphonates for osteoporosis [27] Fracture risk

$\mathrm{PL}=7 \%, \mathrm{D}=4 \%, \mathrm{ARD}=3 \%, \mathrm{PRR}=52 \%, \mathrm{~N}=7, \mathrm{n}=18576$

Antidepressants for major depressive disorder[12] $\%$ responders

$P L=37 \%, D=54 \%, A R D=17 \%, P R R=43 \%, N=142, n=27127$

Anticholinergics for overactive bladder [28] 24 hours micturitions $\mathrm{MD}=-0.68, \mathrm{~N}=12, \mathrm{n}=5977$

Statins for cholesterol lowering [15] Major cardiovascular events $P L=18 \%, D=14 \%, A R D=4 \%, P R R=21 \%, N=14, n=90056$

Aspirin for prevention of vascular disease[11] Secondary prevention of serious vascular events $\mathrm{PL}=8.2 / \mathrm{y}, \mathrm{D}=6.7 / \mathrm{y}, \mathrm{ARD}=1.5 \% / \mathrm{y}, \mathrm{PRR}=19 \% / \mathrm{y}, \mathrm{N}=16, \mathrm{n}=17000$

\section{Effect size \\ $\operatorname{SDM}(95 \% \mathrm{Cl}) /$ AMSTAR}

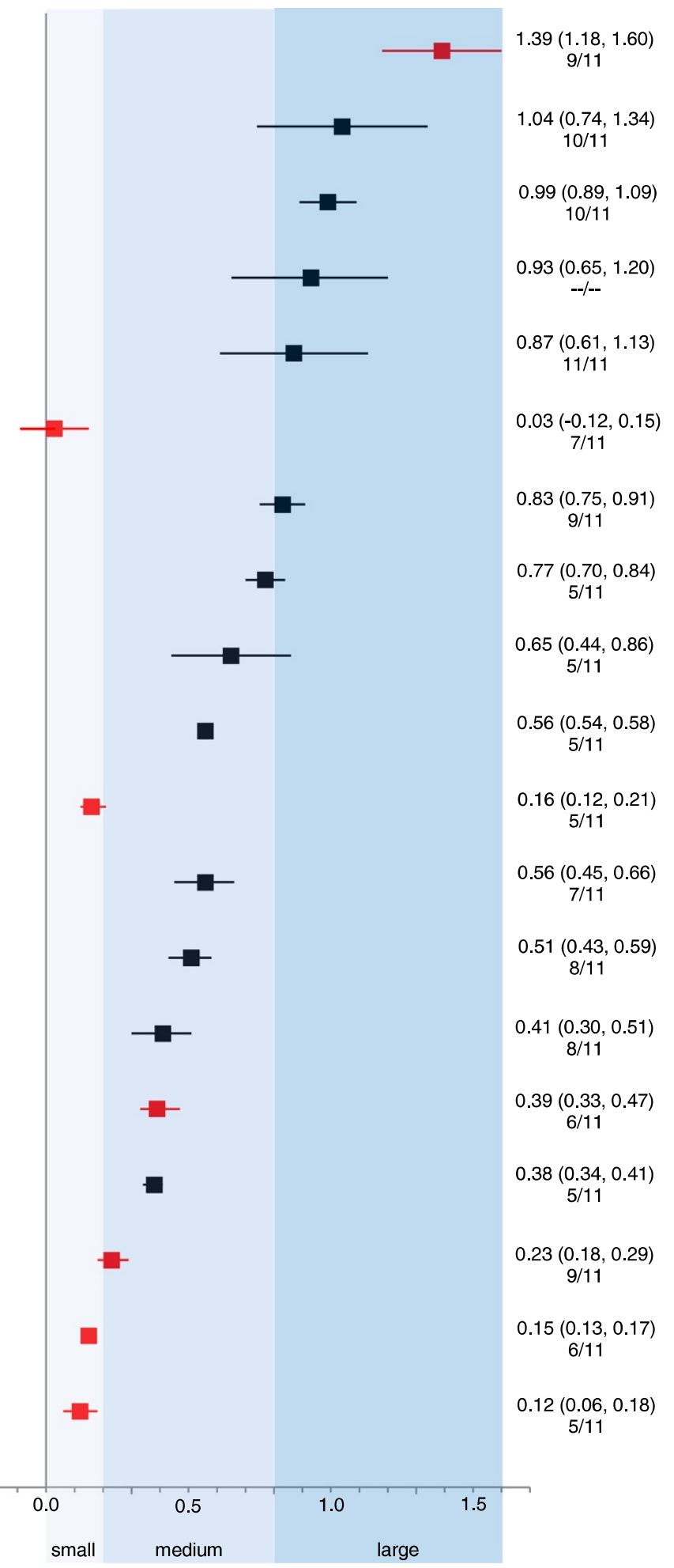

Fig. 1 (See legend on next page.) 
(See figure on previous page.)

Fig. 1 Summary of effect sizes for common pharmacological treatments. The figure presents primary pharmacological intervention for a given therapy type, the primary outcome, descriptive statistics and efficacy measures. Effect sizes are expressed as standardized mean difference with corresponding confidence intervals on the right side and the AMSTAR score below. The graph in the middle shows a ranking of effect sizes according to Cohen: small effect size is no bigger than 0.2; medium effect size is around 0.5; and large effect sizes are bigger than 0.8. Marked with red color are outcomes that can be objectively measured and are patient-oriented [8-12, 15-18, 23-32]. The following drugs listed by the IMS Institute report were not included in the figure: thyroid preparations (no meta-analysis was found); anti-epileptics (no meta-analysis on monotherapy was found because current antiepileptic trials are add-on); hormonal contraceptives for birth control (no "disease" as an indication); and alpha-adrenergic antagonists for benign prostate hyperplasia (no SMD was provided or calculable). All values are statistically significant (except mortality for metformin). All additional confidence intervals can be obtained from the authors upon request. AMSTAR, a measurement scale for the assessment of the methodological quality of systematic reviews; ARD, absolute risk or response difference; $\mathrm{Cl}$, confidence interval; $\mathrm{D}$, percentage of patients with the outcome in the drug group; $\mathrm{MD}$, mean difference in original units; $n$, number of participants; $N$, number of trials; $P L$, percentage of patients with the outcome in the placebo group; PRR, percentage response ratio; SMD, standardized mean difference

number of patients with major cardiovascular events from $18 \%$ to $14 \%$ [15]. The relative risk reduction of $21 \%(100 \%-(14 \% / 18 \%)=21 \%)$ is more impressive than the ARD of $4 \%(14 \%-18 \%=|-4 \%|)$. Findings consistently show that a mere reporting of a relative risk reduction can be misleading, because many clinicians will interpret it as an absolute difference [19].

\section{Limitations}

There are many limitations in an overview of metaanalyses [4]. For example, the meta-analyses differed in methods and publication year. We preferred reviews of drug classes which may obscure superiorities of single drugs. Many outcomes may accumulate over time if the studies had longer durations. For example, the evidence on mortality reduction by statins is based on 5-year studies, but the effect could get larger if patients took them for 20 years. Or a patient with depression may have ten episodes in his life which could be reduced by medication to five [20]. Finally, whether the increment of improvement by a drug is important depends on many factors, such as the seriousness of the disease, side-effects, cost and, most importantly, the short- and long-term outcome in question. For mortality, the "baseline risk" (that is, mortality in the no-treatment group) is often low, leading to a relatively low maximally possible absolute risk reduction. For example, within 5 years without treatment only 9.7/100 participants with hypercholesterolemia died [15], limiting the maximally possible absolute mortality reduction to $9.7 \%$. Nevertheless, since mortality is such an important outcome, even a small reduction can be clinically meaningful. In other words, a large effect size for a transitory rash is less important than a small reduction of death. For all these reasons, this article is only a perspective and not a full review of the evidence for every possible aspect.

\section{Conclusions}

We feel that we need to be more realistic about drug efficacy. Doctors may believe that all patients respond to drugs and none to placebo, but neither statement is true because there is no ideal drug and many disorders remit spontaneously due to their natural course. Our preference for black or white over shades of grey is convenient but it can offer only a "false clarity" [21]. The psychologist Daniel Kahneman received the Nobel Prize in economics for research on cognitive bias and decision making, seen in the context of an initial perception of an idea, which takes place in less than a second versus the more logical thinking through of ideas, which often takes hours or days [22]. The initial rapid intuitions can be biased by many factors such as recency, frequency and vividness of prior personal experiences, but does not take into account statistics very well. Pharmaceutical company advertising takes full advantage of this. We feel these quantitative benchmarks will help clinicians learn how to interpret the latest drug findings and reflect on their limitations. We do not strive to therapeutic nihilism, but rather believe that drug data is complex and requires thoughtful consideration regarding which medications and therapies are best suited for certain situations and patients.

\section{Additional files}

Additional file 1: Details and results of the search. (PDF $257 \mathrm{~kb}$ )

Additional file 2: Study protocol. (PDF $161 \mathrm{~kb}$ )

\section{Abbreviations}

AMSTAR: A measurement scale for the assessment of the methodological quality of systematic reviews; ARD: Absolute response or risk difference; $\mathrm{Cl}$ : Confidence interval; D: Percentage of patients with the outcome in the drug group; MD: Mean difference in original units; n: Number of participants; $\mathrm{N}$ : Number of trials; PL: Percentage of patients with the outcome in the placebo group; PRR: Percentage response ratio; SMD: Standardized mean difference.

\section{Competing interests}

$\mathrm{SL}$ has received honoraria for consulting/advisory boards from Alkermes, Eli Lilly, Janssen, Johnson \& Johnson, Lundbeck, MedAvante, Roche, Otsuka and Teva; lecture honoraria from AstraZeneca, Bristol-Myers Squibb, Eli Lilly, Janssen, Johnson \& Johnson, Lundbeck (Institute), Pfizer, Sanofi-Aventis, ICON, AbbVie, AOP Orphan and Servier; for the preparation of educational material and publications from Lundbeck Institute and Roche; and Eli Lilly has provided medication for a trial with SL as the primary investigator. BH, GG and JMD have no conflicts of interest. This research received no specific grant from any funding agency in the public, commercial or not-for-profit sectors. 


\section{Authors' contributions}

$S L$ and JMD had the original idea for the study. SL and BH identified the eligible meta-analyses, extracted and analyzed the data. GG and BH designed the figure. SL, JMD and BH drafted the manuscript and the figure. All authors revised the manuscript critically for content. All authors read and approved the final version of the manuscript.

\section{Authors' information}

SL is Professor and Vice Chairman of the Department of Psychiatry and Psychotherapy, Technical University Munich, Germany; Honorary Professor of Evidence-based Psychopharmacological Treatment at the University of Aarhus, Denmark; and Editor of the Cochrane Schizophrenia Group. BH is a researcher in evidence-based medicine at the Department of Psychiatry and Psychotherapy, Technical University Munich, Germany. GG is Head of Department for Evidence-based Medicine and Clinical Epidemiology at the Danube University, Krems, Austria; and Associate Director of the Research Triangle - University of North Carolina Evidence-based Practice Center in Chapel Hill, NC, USA. JMD is Research Professor of Medicine at the University of Illinois at Chicago, IL, USA; and Editor of the Cochrane Schizophrenia Group.

\section{Acknowledgements}

We would like to thank Dawn R Gartlehner for proofreading of the manuscript

\section{Author details}

'Department of Psychiatry and Psychotherapy, Technische Universität München, Klinikum rechts der Isar, Ismaninger Straße 22, 81675 Munich, Germany. ${ }^{2}$ Department for Evidence-based Medicine and Clinical Epidemiology, Danube University Krems, Krems an der Donau, Austria. ${ }^{3}$ RTI-International, Research Triangle Park, NC, USA. ${ }^{4}$ Department of Psychiatry, University of Illinois at Chicago, Chicago, IL, USA.

Received: 28 May 2015 Accepted: 18 September 2015

Published online: 02 October 2015

\section{References}

1. Bastian H, Glasziou P, Chalmers I. Seventy-five trials and eleven systematic reviews a day: how will we ever keep up? PLoS Med. 2010;7:e1000326. doi:10.1371/journal.pmed.1000326.

2. Gigerenzer G, Muir Gray J. Better doctors, better patients, better decisions: Envisioning health care 2020. Cambridge, MA: MIT Press; 2011.

3. Lenzer J. Unnecessary care: are doctors in denial and is profit driven healthcare to blame? BMJ. 2012;345:e6230. doi:10.1136/bmj.e6230.

4. Leucht S, Hierl S, Kissling W, Dold M, Davis JM. Putting the efficacy of psychiatric and general medicine medication into perspective: review of meta-analyses. Br J Psychiatry. 2012;200:97-106. doi:10.1192/bjp.bp.111.096594.

5. Aitken M, Kleinrock M, Lyle J, Caskey L. Medicine use and shifting costs of healthcare. A review of the use of medicines in the United States in 2013. Danbury, CT: IMS Institute for Healthcare Informatics; 2014.

6. Cohen J. Statistical power analysis for the behavioral sciences. 2nd ed. Hillsdale, NJ: Lawrence Erlbaum Associates; 1988.

7. Shea BJ, Hamel C, Wells GA, Bouter LM, Kristjansson E, Grimshaw J, et al. AMSTAR is a reliable and valid measurement tool to assess the methodological quality of systematic reviews. J Clin Epidemiol. 2009;62:1013-20. doi:10.1016/ j.jclinepi.2008.10.009.

8. Khan M, Santana J, Donnellan C, Preston C, Moayyedi P. Medical treatments in the short term management of reflux oesophagitis. Cochrane Database Syst Rev. 2007;2:CD003244. doi:10.1002/14651858.CD003244.pub2.

9. Moore RA, Derry S, McQuay HJ, Wiffen PJ. Single dose oral analgesics for acute postoperative pain in adults. Cochrane Database Syst Rev. 2011;9:CD008659. doi:10.1002/14651858.CD008659.pub2.

10. Law M, Morris JK, Jordan R, Wald N. Headaches and the treatment of blood pressure: results from a meta-analysis of 94 randomized placebo-controlled trials with 24,000 participants. Circulation. 2005;112:2301-6. doi:10.1161/ CIRCULATIONAHA.104.529628.

11. Antithrombotic Trialists C, Baigent C, Blackwell L, Collins R, Emberson J, Godwin J, et al. Aspirin in the primary and secondary prevention of vascular disease: collaborative meta-analysis of individual participant data from randomised trials. Lancet. 2009;373:1849-60. doi:10.1016/S01406736(09)60503-1.
12. Undurraga J, Baldessarini RJ. Randomized, placebo-controlled trials of antidepressants for acute major depression: thirty-year meta-analytic review. Neuropsychopharmacology. 2012;37:851-64. doi:10.1038/npp.2011.306.

13. Norman GR, Sloan JA, Wyrwich KW. Interpretation of changes in healthrelated quality of life: the remarkable universality of half a standard deviation. Med Care. 2003;41:582-92. doi:10.1097/01.MLR.0000062554.74615.4C.

14. Law MR, Wald NJ, Rudnicka AR. Quantifying effect of statins on low density lipoprotein cholesterol, ischaemic heart disease, and stroke: systematic review and meta-analysis. BMJ. 2003;326:1423. doi:10.1136/bmj.326.7404.1423.

15. Baigent C, Keech A, Kearney PM, Blackwell L, Buck G, Pollicino C, et al. Efficacy and safety of cholesterol-lowering treatment: prospective metaanalysis of data from 90,056 participants in 14 randomised trials of statins. Lancet. 2005;366:1267-78. doi:10.1016/S0140-6736(05)67394-1.

16. Turnbull F, Blood Pressure Lowering Treatment Trialists' Collaboration. Effects of different blood-pressure-lowering regimens on major cardiovascular events: results of prospectively-designed overviews of randomised trials. Lancet. 2003;362:1527-35.

17. Saenz A, Fernandez-Esteban I, Mataix A, Ausejo M, Roque M, Moher D. Metformin monotherapy for type 2 diabetes mellitus. Cochrane Database Syst Rev. 2005;3:CD002966. doi:10.1002/14651858.CD002966.pub3.

18. Boussageon R, Supper I, Bejan-Angoulvant T, Kellou N, Cucherat M, Boissel $J P$, et al. Reappraisal of metformin efficacy in the treatment of type 2 diabetes: a meta-analysis of randomised controlled trials. PLoS Med. 2012;9:e1001204. doi:10.1371/journal.pmed.1001204.

19. Covey J. A meta-analysis of the effects of presenting treatment benefits in different formats. Med Decis Making. 2007;27:638-54. doi:10.1177/ 0272989X07306783.

20. Glue P, Donovan MR, Kolluri S, Emir B. Meta-analysis of relapse prevention antidepressant trials in depressive disorders. Aust N Z J Psychiatry. 2010;44:697-705. doi:10.3109/00048671003705441.

21. van Deemter K. Not exactly: In praise of vagueness. Oxford: Oxford University Press; 2010.

22. Kahneman D. Thinking fast and slow. New York, NY: Farrar, Straus and Giroux; 2011.

23. Birks J. Cholinesterase inhibitors for Alzheimer's disease. Cochrane Database Syst Rev. 2006;1:CD005593. doi:10.1002/14651858.CD005593.

24. Faraone SV, Buitelaar J. Comparing the efficacy of stimulants for ADHD in children and adolescents using meta-analysis. Eur Child Adolesc Psychiatry. 2010;19:353-64. doi:10.1007/s00787-009-0054-3.

25. Holbrook AM, Crowther R, Lotter A, Cheng C, King D. Meta-analysis of benzodiazepine use in the treatment of insomnia. CMAJ. 2000;162:225-33.

26. Leucht S, Arbter D, Engel RR, Kissling W, Davis JM. How effective are second-generation antipsychotic drugs? A meta-analysis of placebocontrolled trials. Mol Psychiatry. 2009;14:429-47. doi:10.1038/sj.mp.4002136.

27. MacLean C, Newberry S, Maglione M, McMahon M, Ranganath V, Suttorp M, et al. Systematic review: comparative effectiveness of treatments to prevent fractures in men and women with low bone density or osteoporosis. Ann Intern Med. 2008;148:197-213.

28. Nabi G, Cody JD, Ellis G, Herbison P, Hay-Smith J. Anticholinergic drugs versus placebo for overactive bladder syndrome in adults. Cochrane Database Syst Rev. 2006;4:CD003781. doi:10.1002/14651858.CD003781.pub2.

29. Sin DD, Man J, Sharpe H, Gan WQ, Man SF. Pharmacological management to reduce exacerbations in adults with asthma: a systematic review and meta-analysis. JAMA. 2004;292:367-76. doi:10.1001/jama.292.3.367.

30. Barr RG, Bourbeau J, Camargo CA, Ram FS. Tiotropium for stable chronic obstructive pulmonary disease: A meta-analysis. Thorax. 2006;61:854-62. doi:10.1136/thx.2006.063271.

31. Derry CJ, Derry S, Moore RA. Sumatriptan (oral route of administration) for acute migraine attacks in adults. Cochrane Database Syst Rev. 2012;2:CD008615. doi:10.1002/14651858.CD008615.pub2.

32. Fahn S, Oakes D, Shoulson I, Kieburtz K, Rudolph A, Lang A, et al. Levodopa and the progression of Parkinson's disease. N Engl J Med. 2004;351:2498-508. doi:10.1056/NEJMoa033447. 